\title{
Matrix-Assisted Pulsed Laser Evaporation (MAPLE) technique for deposition of hybrid nanostructures
}

\author{
Songlin Yang ${ }^{1}$ and Jin Zhang ${ }^{*}$ \\ ${ }^{1}$ Department of Chemical and Biochemical Engineering, University of Western Ontario, London, Ontario, Canada
}

\begin{abstract}
This review paper provides the detailed information on a new nano-manufacturing process for producing nanocomposites, i.e., the matrix-assisted pulsed laser evaporation (MAPLE). Owing to its unique advantages in depositing polymers and biomaterials, MAPLE technique has been applied to fabricate coatings for medical implants, and electronic devices. In this paper, the current progress on the applications of MAPLE technique is reviewed and discussed. MAPLE techniques have demonstrated a precise control of the parameter such as film thickness and roughness, material structure after deposition. The potentials of MAPLE technique in nanobiotechnology are also discussed in this review paper.
\end{abstract}

\section{Introduction}

The key of development of the next generation electronic devices, passivation coatings chemical and biological sensors. For these applications, their chemical composition, structure and morphology should be in a definite area. These application include organic thin-film transistors, organic light emitting displays, flexible optical waveguides, nonlinear and optical limiting devices and other opto-electronic components [1]. The organic and inorganic material thin film also is an important part of high performance dielectrics, optical data storage, passivation and encapsulation coatings for electronic devices. At pharmaceutical, bioengineering and sensing field, the chemoselective or bio-specific layers are based on the polymer and organic thin film which make the most types of chemical and biochemical sensor system in operation. The biomaterial thin film is the essential part of tissue engineering, spatial patterning of cells, time-release drug delivery systems, anti-inflammatory coatings for medical implants and implantable devices, in biomedical field.

A new deposition technique, known as Matrix assisted pulsed laser evaporation (MAPLE) was designed for providing an alternative way to fabricate polymeric and organic thin film. In the following sections origins, development process and the application of MAPLE technique will be discussed and the overview of presenting understanding of process will be presented together. Following by the conclusion of this technique and outlook to the future of MAPLE.

\section{Background}

Surface modification refers to modifying the surface of a material by altering its surface properties to enhance specific functions while retaining the bulk properties of the desired material (by fabricating thin film on material's surface). The modification can be done by different methods to alter a wide range of characteristics of the surface, such as: roughness [2], hydrophilicity [3], surface charge [4], surface energy, biocompatibility [5] and reactivity [6].

There are two main type of surface modification methods: chemical methods and physical methods. The two main chemical methods are chemical vapor deposition(CVD) [7] and wet chemical methods [8].
CVD method is use to increase the hydrophilicity of a surfaces by adding suitable functional groups. However, the precursors of CVD method can be highly toxic, corrosive or explosive $\left(\mathrm{Ni}(\mathrm{CO})_{4}, \mathrm{SiCl}_{4}, \mathrm{~B}_{2} \mathrm{H}_{6}\right)$. These compounds may cause damage of the biomaterial [9]. And there are a series of by products ( $\mathrm{CO}, \mathrm{HF}$ or $\mathrm{H}_{2}$ ) may produce by the CVD process. Same with the CVD method, wet chemical method involve chemical agents among the process which can cause adverse toxic effects. Besides, the chemical methods strongly depend on the use of surfacespecific chemistry. This is the reason which obstructed the chemical method to be employed to modify a wide range of substrates [10].

Physical methods include spin coating [11], dip coating [12] and physical vapor deposition (PVD)[13]. Compare to the chemical method, spin coating and dip coating are more eco-friendly. But these method are hard to control the thickness of the film compare to the PVD method. For the PVD method, the thickness of film can be control to atomic level or nanometer level and the solvent contamination is much lower. The PVD method include vacuum evaporation, sputtering, arc vapor deposition and ion plating [9]. These four method have their own advantages and disadvantages, and can be applied only to specific range of materials.

Matrix assisted pulsed laser evaporation (MAPLE) is a new deposition technique which is developed from pulsed laser deposition (PLD). MAPLE provides a gentle process for fabricating a uniform film of small or large molecular weight species such as inorganic and polymers, from the condensed phase into the vapor phase.

For the process of MAPLE technique, a frozen target consisting of a solution of a polymeric compound dissolved in a relatively volatile solvent and it would be ablation by the laser among the deposition process. The target material is dissolved in the solvent with a weight concentration lower than $5 \%(\mathrm{w} / \mathrm{v})$. During the deposition process,

Correspondence to: Dr. Jin Zhang, Department of Chemical and Biochemical Engineering, University of Western Ontario, London, Ontario, Canada

Received: April 24, 2017; Accepted: May 15, 2017; Published: May 19, 2017 
the majority of the laser energy is initially absorbed by the solvent molecules. This mechanism can protect the target molecules and prevent them from being damaged by the high energy laser beam. Among the photo thermal process, the frozen solvent molecules absorbed the energy of photons and converted to thermal energy which in turn heats the target molecules and allows the solvent to vaporize [14]. When the target molecules absorb enough energy through collisions with solvent molecules under the evaporation process, the target molecules are transferred to the vapor phase. By the MAPLE process, the target solution (matrix) is, layer by layer, depleted and maintain the same concentration. The substrate is placed on the direction of the molecule movement to make the target molecule reach it easily. During the MAPLE process, the target molecules start fabricating the thin film on the surface of substrate. At the meantime, the solvent molecules are pumped away by the pumps because their adhesion coefficient are low [15].

\section{The history of MAPLE}

The first organic and polymeric materials deposition were finish by Smith and Turner through PLD method [16]. The material they deposited is called fuchsine and Ni-dimethyl glyoxime (organic dye). After 2 decades, Hansen and Robitaille tried to deposit polymer (polyethylene, polycarbonate, polyimide and polymethylmethacrylate) film by PLD method $[17,18]$. During the experiment the researchers found that as the laser wavelength decreased, wavelength the film morphology and the ablation behavior improved. And the other observation was the film quality was enhanced when laser energy close to the ablation threshold energy of the polymers.

Even though there are many of polymers and organic materials deposited by the PLD, there still have problem that PLD method hard to avoid. During the PLD process, the organic or polymeric targets are directly ablated by the high energy pulse laser. This may lead to the damage of the structure or degree of irreversible decomposition.

Since PLD is not always suitable for depositing delicate materials like polymers and organic materials, other laser based deposition methods are considered. The MAPLE technique was developed by the US Naval Research Laboratory to provide gentler pulsed laser evaporation process for functionalized polymers in the late 1990s [19]. The MAPLE technique has achieve success at depositing thin and uniform layers organic compounds such as simple carbohydrates and their polymers $[15,20]$. Compare to PLD method, MAPLE technique give the researchers a milder choice to deposit materials through pulse laser deposition process. The target materials dissolved in the highly volatile solvent and the solution which maintained frozen by the liquid nitrogen. Most of the laser beam energy would be absorbed by the solvent and the target material can be protected as this paper discuss before. The MAPLE technique is able to fabricate homogeneous, ultra-thin, well adherent coatings over desired substrate with accurate thickness control, and maintains the chemical structure and the physiochemical properties of the organic or polymer molecules $[21,22]$.

\section{The MAPLE technique}

Among MAPLE process, the concentration of the desired solute material (i.e., polymer or biomaterial to be deposited) in the target solution always lower than $5 \%$. Therefore, each molecule of target material is surrounded and shielded by a large amount of solvent molecules (matrix molecules). The mechanism can prevent the target molecules (polymer or biomaterial molecules) from the thermal damage during the laser ablation. Under this condition, the highlyvolatile solvent molecules absorb the laser radiation energy and fracture away from the surface of the frozen target.
A typical MAPLE system is sketched in Figure 1 (the vertical configuration). The whole process is carried inside a vacuum chamber. The substrate holder fixed on the top of the chamber. The soild substrate is fixed on the surface of the holder. On the opposite side of the subtrate holder is the target holder. The target solution is add inside the target holder and freezed by the liquid nirtrogen. The structure of the target holder is complex than the substrate holder, the wall of the target holder is double layer and the liquid nitrogen run through to freeze the target solution. Both these two holders are rotating during the deposition process to fabricate thin film with better morphology and make sure that the laser ablate the most of the target surface. The high energy laser beam come through the focusing and the $\mathrm{CaF}_{2}$ glass and heat the surface of the frozen target. Normally, the background pressure of the chamber is around $1 \times 10^{-4}$ Torr.

When the deposition process start, the target solution with desired solute material (low concentration $<5 \%$ ) inside is frozen by the liquid nitrogen first. When the target is frozen, the chamber is pumped down by the vacuum pump. The high-energy pulse laser beam impinging on the target surface and remove the molecules from the target surface. Normally, the solvent molecules are lighter than the target material molecules, it would be easily pumped away by the vacuum pump. Only the latter molecules which is heavier could reach the substrate surface. Therefore the presence of the solvent minimizes the photochemical damage of the target material molecules.

Figure 2 is the sketch of the horizontal configuration. The mechanism of horizontal configuration is same with vertical configuration. The different between them is the location of the target holder and substrate holder. For the vertical configuration, the substrate holder placed opposite in a vertical direction. But for the horizontal configuration, the substrate holder placed opposite in a horizontal direction.

But to achieve this result, the solvent (matrix) need to meet three specific criteria. First, the target material must be highly soluble in the solvent. Second, the most part of the laser beam energy must mainly be absorbed by the solvent. Third, the solvent (matrix) must be highlyvolatile at room temperature. As we disscuss before, the target and substrate are oriented with respect to each other (both vertical and horizontal). The lifting target material could be deposited on the surface of the soild substrate. When the process start, the high-energy pulse laser hit the surface of the frozen target and initiate the photothermal proces, this process sublimate the frozen solvent and release the coating material into the vacuum. The result of this process is the solvent molecules and the particles would gain momentum and moving from the frozen target to the substrate. As the solvent is light and its vapor pressure is high at room temperature, it would be removed by the vacuum pump. Meanwhile, the target material molecules which is lighter adhere on the surface of the substrate and fabricate thin film.

\section{MAPLE deposition}

\section{Early Success by MAPLE}

The early experiments of MAPLE are mainly focus on the polymers. From the very beginning, there are two primary targets: biological molecules and polymers. For example, the carbohydrates, chemoselective polymer and flouroalcoholpolysilane. Initially, Naval Research Laboratory deposited carbohydrates like glucose, sucrose, and dextran $[15,23]$ Fluoro alcohol Polysiloxane.

(SXFA), the film which deposited by MAPLE shows highly sensitivities to chemical threatment than the spray coated thin film $[15,24,25]$. The following figure shows of two surface acoustic wave 


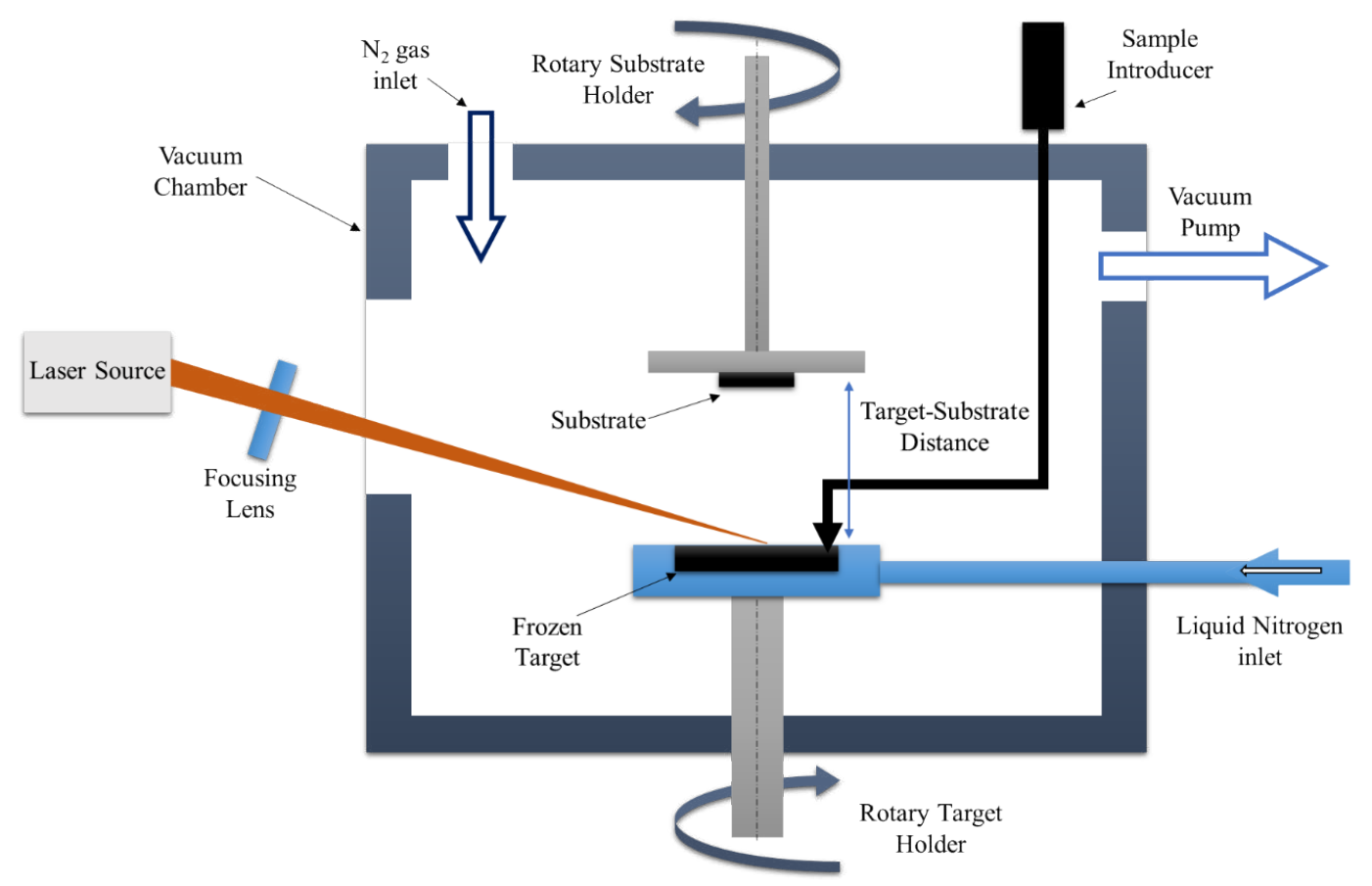

Figure 1. Schematic of MAPLE (verticalconfiguration)

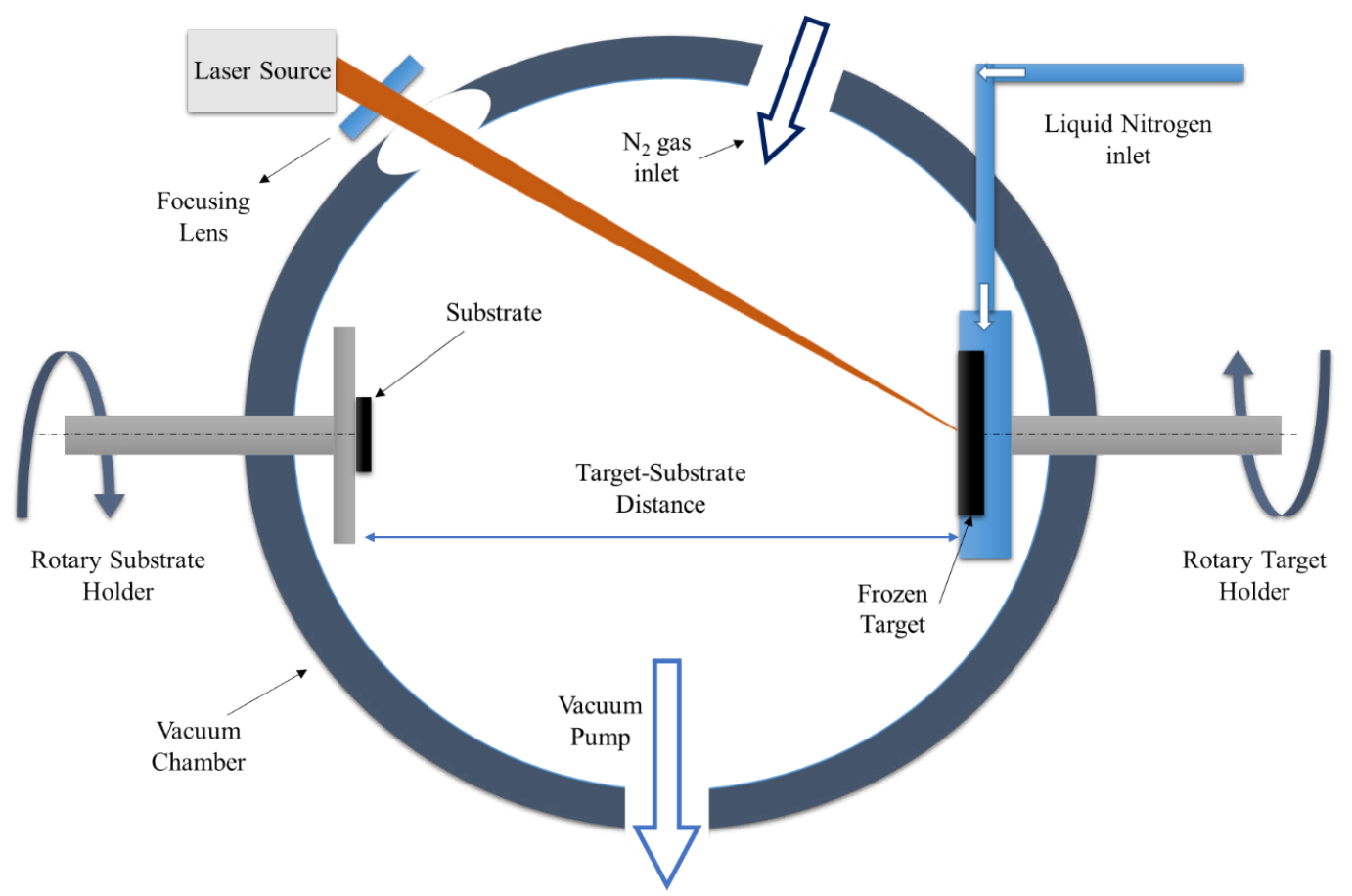

Figure 2. Schematic of MAPLE (horizontalconfiguration)

(SAW) devices, the film which fabricated by MAPLE (a) is more uniform, compared with spray coating one(b). The FT-IR result of the MAPLE deposited SXFA film present that the spectra was identical to that of bulk SXFA samples and with the similar absorbance ratios for the key absorption bands. In order to get desired sensor signal kinetics, it may need to get the thickness of the film lower than 50 $\mathrm{nm}$ and make its surface highly uniform. The AFM result show that the thin film fabricate by MAPLE meet this requirement better than the other methods.
The deposition of horseradish peroxidase and insulin by Ringeisen [26] demonstrated that the MAPLE process can avoid the destruction of the structure and preserved the activity of the biomaterial among the process. Also $\mathrm{Wu}$ [27] conduct the experiment to deposit horseradish peroxidase in a polymer composite for biosensor applications. The researchers also conduct the experiment about deposit polymer by MAPLE for organic electronic devices and sensors. For the light emitting diodes application some polymers such as MEH-PPV and poly(2-methoxy-5-(2'-ethylhexyloxy)-p-phenylene vinylene) were 

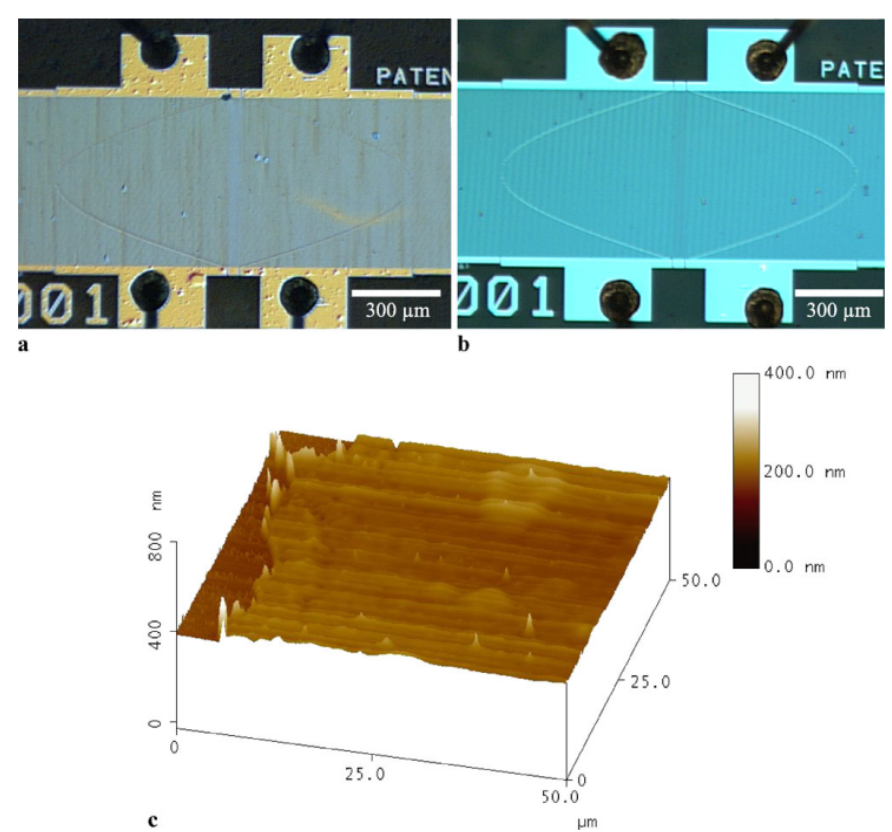

Figure 3. Optical micrographs showing the surface of SAW devices coated with a chemoseletive polymer (SXFA) by (a) spray coating (b) MAPLE (c) AFM image of the film. Reproduced with permission. ${ }^{1}$ Copyright 2017, Springer

deposited by the researchers [28]. Another research focus is depositing polythiophene polymer thin film to fabricate the active layer for organic photovoltaic devices [21-29]. For the sensor application studies, polysiloxanes and polysiloxanes were deposited, because those can use as transparent electrode material [30-32]. Even polymer nanocomposites film was fabricated and it doped with carbon nanotube which is conductive were deposited. This doping material may can help increase the electrical conductivity and mechanical properties of the polymer thin film $\mathrm{n}$ [33-34].

\section{Recent progress of MAPLE}

\section{Biomaterials}

Biomaterials include a large range of materials. From simple polymers for example poly(lactic acid) (PLA) for tissue engineering to nucleic acids and proteins, biomaterials can be applied in medical applications, textiles, agriculture and food industries. The biomaterials are different with the traditional materials like metals because it possess inherent activity (biochemical functions). But for the most of the biomaterials, the denaturing may change it property. Normally, it's easy to denature the biomaterials, it may happen during the treatment process. Besides, the mass and the size of the bimaterial molecules are also important for the biomaterials properties. Therefore it's necessary for the thin film fabricating process to preserve the size, mass and structure of the biomaterial molecules among the depostion process.

Serval studies about biomaterial deposition are discussed in the former section. Recent years serval bio-compatible polymers were deposited by MAPLE. For example, collagen [35], the copolymer like poly(d,l-lactide-co-glycolide) (PLGA) [36] and polyethylene glycol (PEG) [37]. For PEG deposition, it was shown that the PLD method may cause the irreversible change of the target materials, due to the photochemical decomposition [37]. On the contrary, the PEG thin film which deposited by MAPLE method almost no change from the bulk, after analyze the result from the FTIR, MALDI-time of flight (MALDITOF) and electrospray ionization (ESI) [37]. Another study show that, it is possible to deposit PEG thin film by high-energy $\left(2.5\right.$ to $\left.7 \mathrm{~J} / \mathrm{cm}^{2}\right)$ when they use water as matrix [38].

As previously discussed, it is possible to deposit protein on the soild substrate by MAPLE process. Some protein like Ribonuclease have potential to inhibit the growth of cancer cells. It is hard to immobilize enzyme on the substrate surface and maintain its enzymatic activity. But this problem can be solved by using the MAPLE process. Popescu deposited thin films of Ribonuclease by MAPLE process [39]. At the sametime, this preserving the chemical structure and enzymatic acivity of the target material. After analyzed the film, they also found special morphologies of this thin film. The nano- and micro-scale particles are consist together on the substrate surface (Figure 4a). Levan as a biopolymer can be use as pharmaceutical excipient, drugdelivery carrier and medical implant coating. Sima et al. deposited the levan coating by MAPLE process [40]. It displayed good compatibility with bone cells. Meanwhile, this levan thin film present a two-dimension special nanostructure: parallel ridges a few hundred $\mathrm{nm}$ in width (Figure $4 \mathrm{~b}$ ). The researchers presumed reason of fabricating this nanostructure was evaporation-induced self-assembly. The above results indicated MAPLE can be useful as a tool to produce complex drug delivery systems.

MAPLE method can be applied in fabricating biological compatible and functional thin film for implant devices of medical field. Gentamicin sulfate, a antibiotic against Staphylococcus aureus, Escherichia coli, and many other bacteria. The co-polymer which mixed by this antbiotic was deposited by MAPLE [41]. This anit-microbial polymer films are used for prevent the infaction caused by the bacteria from implants and catheters. The surface morphology of the MAPLE deposited antimicrobial film maybe rough but it is continuous. The researchers would control the roughness to improve performance of MAPLE-deposited medical films in the future, by controling the laser parameters and the MAPLE parameters. Similar thin film for medical inplant were deposited by Miroiu et al. [42] using MAPLE technique. They fabricate thin films by using hydroxyapatitesilk fibroin composite. This thin film possess a series of special properties like high tensile strength and elasticity because this material combined the bone-like characteristics of hydroxyapatite.

The MAPLE can fabricate versatile and durable thin film coating by hydroxyapatitesilk fibroin composite because the solvent(matrix) can protect the fragile silk fibroin molecules from the damage of the high energy laser beam. Floroian et al. [43] report a new biomaterial thin film deposited by MAPLE technique for cell adhesion. The researchers mixed bioactive glass (BG57) with PMMA polymer to interaction

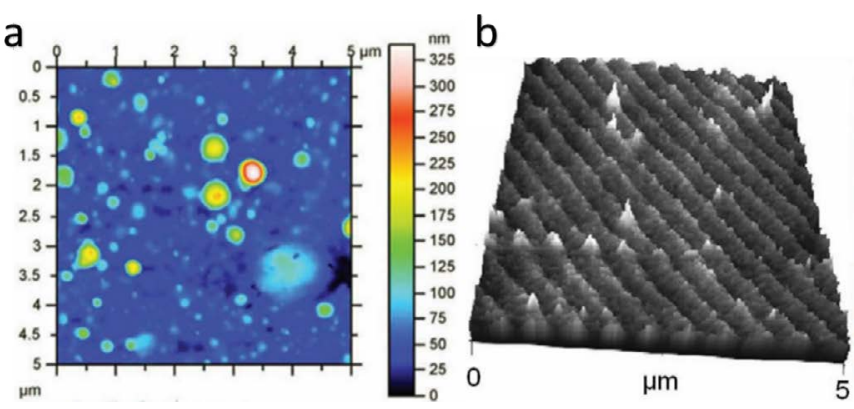

Figure 4. a) AFM height image depicting the nanoscale surface morphology of MAPLE deposited Ribonuclease. Reproduced with permission ${ }^{39}$. Copyright 2017, Cambridge University Press. b) AFM representation of the structured surface morphology exhibited by MAPLE-deposited oxidized levan.Reproduced with permission ${ }^{40}$. Copyright 2017, American Chemical Society. 
between implant and bone. Because the bioactive glass was brittle so the researchers introduce the PMMA inside of the thin film material to improve the mechanical properties. The researcher deposited this thin film on the surface of titanium implants surface in order to prevent the onization of the titanium metal which need to embed into the body environment. MAPLE method also provide another way to produce coatings for the proteins which are hard to immobilize on the surface of the substrate. But this series of proteins may can improve the cell adhesion of the surface of the medical implants. Recently year, the researchers were successful deposited the thin films of fibronectin [22] and vitronectin [44] by MAPLE method. Same with the other experiments, because the MAPLE process can preserve the properties and the structure of the protein, human osteoprogenitor cells which grown on the film presented good adhesion on its surface. A lot of reports mentioned using MAPLE technique to deposit Poly(ethylene glycol) (PEG) as biocompatible thin film. An experiment was carried out by Paun et al. [45] to compare the blood compatibility, morphology and surface properties of MAPLE deposited different moleculer weight PEG films. This study indicate that PEG was more compatible with blood and present low hemolysis property at the highest moleculer weight $(10 \mathrm{kDa})$ of this study. MAPLE method suit to deposit delicate material especially the biomaterial because its molecule structure is easy to break.

\section{Polymers}

In this part, the discussion is focusing on the polymers deposited for electronic devices and sensors. In recent year, the MAPLE method was used to deposit conductive polymer in order to fabricate electronic devices. Since the films deposited by MAPLE method are ultra-thin and uniform and it is not hard for MAPLE method to achieve complex layer structure. For the early experiment, the MEH-PPV was deposited by MAPLE method use IR-laser sourse $(2940 \mathrm{~nm})$. But the problem was almost $50 \%$ of the molecular weight reduced after deposition [46]. And some researchers deposited light-emitting polymers like polyfluorene (PFO). Both UV [47] and IR [48] laser source can be applied to deposit PFO. MAPLE process can minimize the degradation of the PFO molecules when appropriate solvent were selected. Though PFO's light emitting property is very sensitive to molecule structure, it still can preserve nice blue light emitting after MAPLE process (Figure 5). The present that its molecular structure were protected during the MAPLE process.

Another new polymer which is used in photovoltaic field is poly(3hexylthiophene) or P3HT. Ryan et al. also studied its MAPLE deposition process [49]. On the other hand, A. P. Caricato and his co-workers

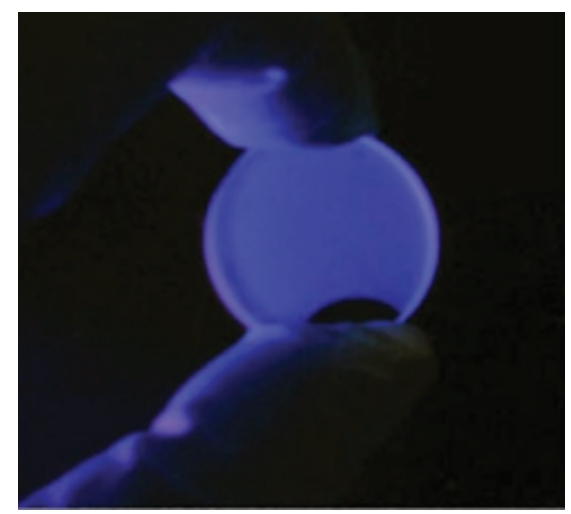

Figure 5.Picture of MAPLE deposted PFO film under the UV-light. Reproduced with permission..$^{48}$ Copyright 2017, Springer. produced a new bilayer photovoltaic device by MAPLE process. The bilayer system were fabricated by [6,6]-phenyl-C61-butyric acid methyl ester (PCBM) and P3HT through a one-step deposition. The highlight of this study is that they used a two component target cup and produce the device by one step [50]. This new method raised a new concept that performing the bilayer depsotion without breaking the vacuum of the equipment. This method can avoid the contaimination of the air when we need to load another layer material. The problem is althrough the bilayer solar cell was operational but the effciency of this device was two orders of magnitude below the normal P3HT/PCBM bilayer cell. Other electronic devices which fabricate by MAPLE were developed in recent years. Field-effect transistors (FETs) were develop by Adil et al. [51] Among the experiment, the $\mathrm{SiO}_{2}$ worked as soild substrate and used MAPLE technique to deposit copolymer 9,9-dioctylfluoreneco-bisN,N-(4-butylphenyl)-bis-N,N-phenyl-1,4-phenylenediamene (PFB) on its surface. The $\mathrm{SiO}_{2}$ layer was served as the dielectric layer and the PFB copolymer layer was served as active layer for the FET device. And the FET device fabricated by MAPLE presented better on/off ratio than the devices produced by spin coating. The same happened with metal-insulatorsemiconductor (MIS) diodes, Ukah et al. [52] carried the similar experiment by MAPLE deposition. Beside, Guha et al. [53] performed MAPLE depsotion experiment to fabricated FET device by P3HT. The result indicated that the FET device which produced by MAPLE deposition was showed comparable performance to the FET device (P3HT) produced by spin coating.

For optoelectronic devices, one of the important things is to control what light is reflected and what light is absorbed. Normally, this aim would be finished by turning the refractive index of the device surface or device coating. It is difficult for all-organic devices to change the refractive index. Because the limited range of refractive indices for standard polymers. MAPLE technique can be applied to solve this problem in this field. Polymer nanocomposites can help to enlarge the range of the refractive indices, just by introduce nanoinclusions into the polymers. In recent years, poly(3,4-ethylenedioxythiophene)poly(styrenesulfonate) (PEDOT-PSS) with gold nanoparticles, copper nanoparticles or single carbon nanowire were deposited by IRMAPLE [48]. After characterization, the researchers found that the nanoparticles form a saturate network with electrical property. The other way to change the refractive index is introducing nanoscale pores which filled by air into the film. The co-depositing of polystyrene (PS) and PMMA was carried by McCormick et al. [54]. After the deposition process, researchers dissolved away the PMMA. And after of the thin film would be replaced by the nanoscale pores. Bargg reflector was fabricated by MAPLE technique by deposited 16 alternating layers of P3HT and PMMA [46].

\section{Nanoparticles}

Recent years, since the nanoparticles became the one the most hot field of material science. Therefore, more and more researchers studied about fabricate thin film by nonoparticles or nanocomposites. The properties of the nanoparticles and nanostructured films were strongly related to surface properies of the nanoparticles, composition, size and morphology. The nanoparticle film can be obtained by metalorganic chemical vapor deposition [55] and molecular beam epitaxy [56]. Compare with MAPLE and PLD, the those two deposition techniques are too delicate, too long to carry out and costing to high. Both MAPLE and PLD could easily control the thickness of the film, compare with some cheaper method like spin coating and drop casting. However, compare with PLD, MAPLE technique has more advantages at protect the structure of the target material. Since most of the nanoparticles 
are delicate and fragile, this is why MAPLE IS considered as a very attractive alternative method than PLD. Wu et al. [57] deposited carbon nanotube film by MAPLE method. And after that success, $\mathrm{TiO}_{2}$ and $\mathrm{SnO}_{2}$ nanoparticles were deposited by the researchers [58]. After characterization, the researchers found that the nanoparticles maintain same crystal structure and size. Angel and his co-works [59] successfully deposited functionalized single wall carbon nanotubes by MAPLE method. The HR-TEM picture (Figure 6) indicate that the structure of the carbon nanotubes were protect by the matrix. In this study, different laser fluence was applied to investigate the relationship between the laser fluence and the material structure (after deposition). The result of this study is that the increase of the laser fluence would lead to the decomposition of the functional groups(carboxylic acid groups) when the laser fluence exceeded $0.4 \mathrm{~J} / \mathrm{cm}$.

In recent years, the researchers have change their focus of the nanoparticle deposition. A series of nanoparticles which have delicate structure were deposited. Hunter et al. [60] deposited carbon nanopreals and fabricated gold/nanoparticle composite films by MAPLE. Magnetron sputtering was applied in this experiment and combined with MAPLE to produce nanocomposite. Gyorgy et al. [61] deposited CdSe/ZnS Core/Shell quantum dots by UV-MAPLE. The HR-TEM image (figure 7) indicate that the crystal structure of the $\mathrm{CdSe} / \mathrm{ZnS}$ quantum dots remain intact.

Angel and his co-workers [62] studied about depositing graphene oxide/iron-oxide nanocomposite by MAPLE method. The nanocomposite films were deposited on the surface of polydimethylsiloxane substrates. The graphene oxide/iron oxide nanocomposites were deposited by two ways: one is vacuum background and another is ammonia rich background. The result show that the formation of pyridinic nitrogen moieties in the basic plane of graphene oxide.

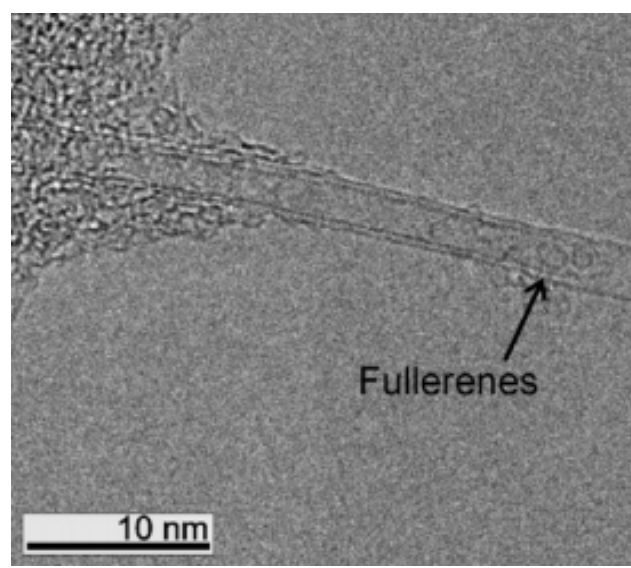

Figure 6. HR-TEM picture of the deposited single wall carbon nanotubes (SWCNTs). Reproduced with permission. ${ }^{59}$ Copyright 2017, Elsevier.

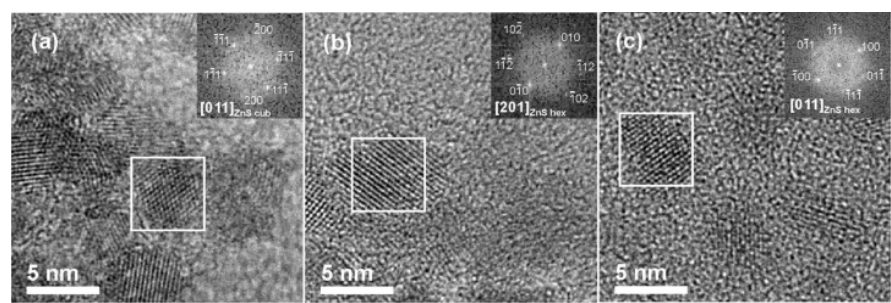

Figure 7. HR-TEM image of CdSe $/ \mathrm{ZnS}$ particles after (a) drop-cost (b) $0.2 \mathrm{~J} / \mathrm{cm}^{2}$ laser fluence (c) $0.1 \mathrm{~J} / \mathrm{cm}^{2}$ laser fluence. Reproduced with permission. ${ }^{61}$ Copyright 2017, American Chemical Society.
At same time, a series of magnetic particles and polymer composites were deposited by the researchers. R. Cristescu et al. [63] deposited $\mathrm{Fe}_{3} \mathrm{O}_{4} /$ oleic acid/cefepime nanoparticles by MAPLE process. Alina et al. fabricated $\mathrm{Fe}_{3} \mathrm{O}_{4} @$ Cinnamomum verum film by MAPLE method. The characterization result show that the property and the structure of the particles were still preserved. The thin film of $\mathrm{Fe}_{3} \mathrm{O}_{4} @$ Cinnamomum verum increase anti-adherent (bacteria) properties of the gastrostomy tubes. Valentina Grumezescu and her co- workers deposited magnetic particles covered by polylactic-co-glycolic acidpolyvinyl alcohol (PLGA-PVA) onto the solid surface. The SEAD patterns of the particles indicated that the deposited particles still keep it property and structure. Though the bioevalution tests, it can be found that the biocompatibility of this film is good and possess anti-adherence and antibiofilm properties. The table one summary the organic and inorganic film which deposited by MAPLE.

\section{Our contribution}

For over a decade, the MAPLE technique was used to modify the surface of the biomaterials by depositing the materials like polymer to change the property of its surface. To overcome the drawback of the biomaterials, various polymers and nanomaterials were chosen by the researchers. Among these drawbacks, irreversible protein adsorption and bacterial contamination on the surface of biomaterials may cause severe problem of the biomaterials and implants. Therefore a lot of the researchers were focusing on this topic and studying prevent this problem by various surface modification methods. Although a lot of works has been published in this field, there were not many researchers focus on surface modification by MAPLE to prevent biofouling, which is specifically suitable for modifying the surface of biomaterials.

Our group has focused on this topic for a long period. The biomaterial which was used to be modified was silicone hydrogel. Silicone hydrogel has better oxygen transfer rate than the conventional hydrogels because it has a different oxygen transport route with less resistant. This transport mechanism is based on the inclusion of siloxane group (Si-O-Si) in the polymeric network [64]. However, the native hydrophobicity and biofouling tendency of silicone hydrogel would strong limiting its biomedical application. Studies show that proteins get adsorbed on the hydrophobic surface within seconds and will cause adverse side effects like microbial infection [65-67]. In order to be used in biomedical applications, surface of silicone hydrogel need to be modified to increase the hydrophilicity of silicone hydrogel. Our group have done several studies about this topic. Pei and his coworkers [68] deposited Pei and his co-workers deposited PEG on the surface of the silicone hydrogel to change the hydrophobic property of it. The result (Figure 8) indicate the antimicrobial efficiency of the silicone hydrogel surface increased since the surface of silicone hydrogel becoming hydrophilic.

Guobang and his co-workers deposited ZnO-PEG nanoparticles by MAPLE method. Zinc-base nanomaterials process excellent anticorrosion property and good antibacterial property. The result show that $\mathrm{ZnO}-\mathrm{PEG}$ nanocomposited coating (Figure 9) reduces over 50\% protein absorption on silicone hydrogel [69].

Meanwhile, our group studied about deposited Ag-PVP nanoparticles MAPLE technique. Guobang and his co-workers deposited Ag-PVP nanoparticles by traditional MAPLE method [70]. The average diameter of Ag NPs that have undergone the MAPLE process for $60 \mathrm{~min}$ is $11.61 \pm 3.58 \mathrm{~nm}$. The low diameter Ag NPs would have more surface area to release the silver ion to increase the antimicrobial efficiency. And the result show that over $60 \%$ of UV light 

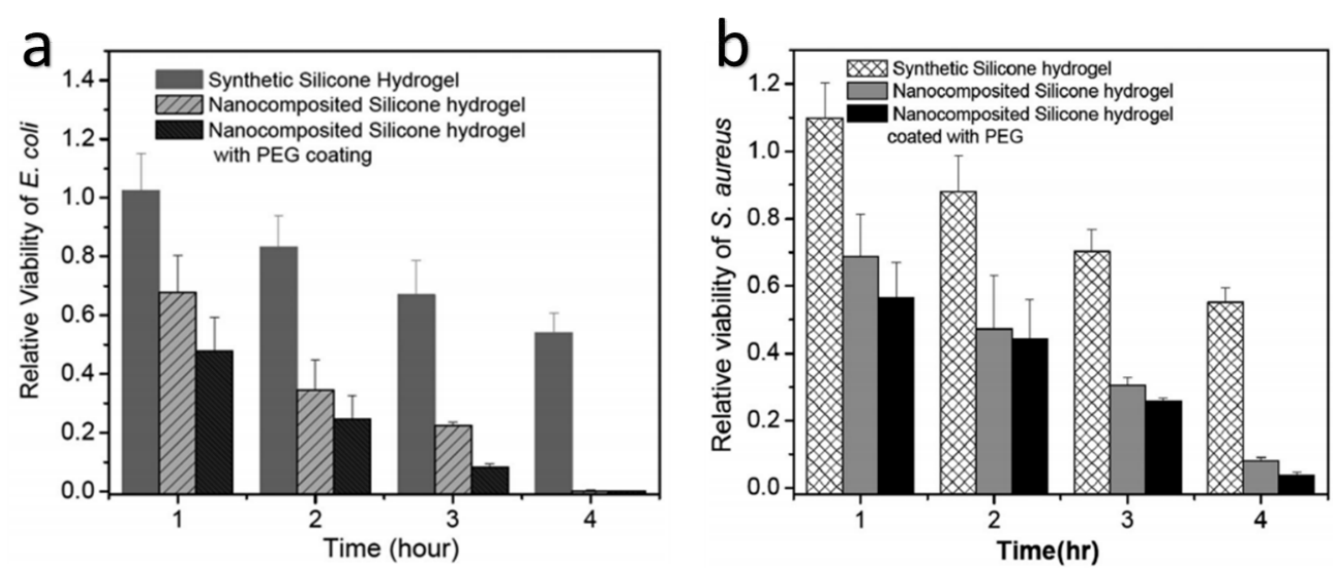

Figure 8. Antimicrobial efficiency of silicone hydrogel and nanocomposited silicone hydrogel with/without PEG coating to (a) E. coli and (b) S. auerus. Reproduced with permission. ${ }^{6}{ }^{6}$ Copyright 2017, Royal Society of Chemistry.

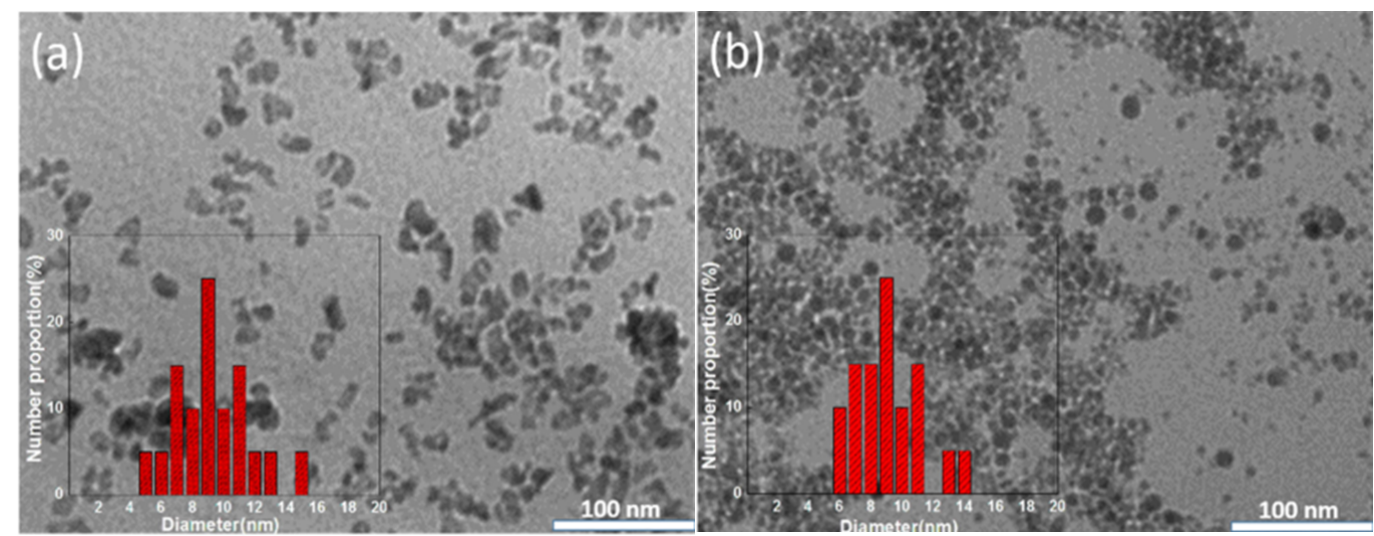

Figure 9. TEM micrographs of (a) ZnO-PEG NPs prepared by sol-gel method, (b) ZnO-PEG NPs deposited on Cu grid by MAPLE process. Reproduced with permission. ${ }^{69} \mathrm{Copyright}$ 2017, Elsevier.

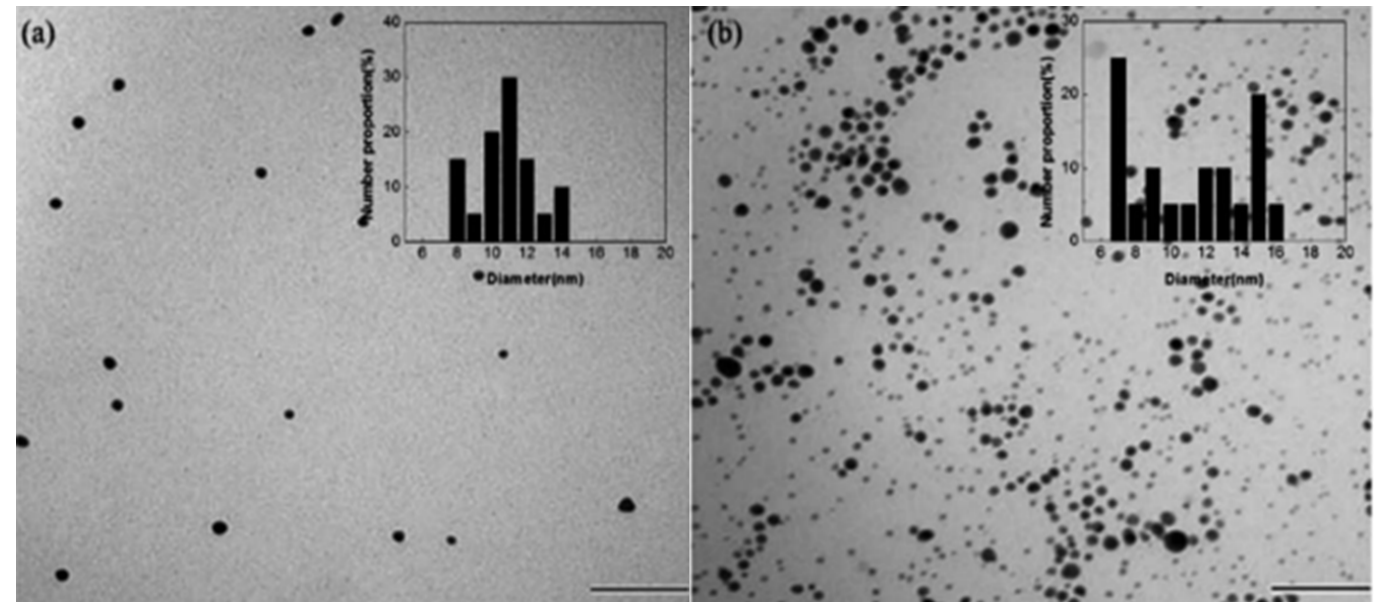

Figure 10. TEM micrograph of (a) Ag NPs produced by photochemical reduction; (b) Ag NPs deposited on a $\mathrm{Cu}$ grid through the MAPLE process. The scale bar refers to $100 \mathrm{~nm}$ Reproduced with permission. ${ }^{70}$ Copyright 2017, Royal Society of Chemistry.

in the range of 300-450 $\mathrm{nm}$ can be blocked by this Ag NPs thin film and the silicone hydrogel still process the high-oxygen permeability.

\section{Future research for MAPLE method}

During reviewing the former works and processing the MAPLE experiment, the problem that the parameter of MAPLE system strongly influence the result of the experiment came out. For example, the wavelength of the laser source for the MAPLE system are vary. (from $193 \mathrm{~nm}$ to $3000 \mathrm{~nm}$ ). But most of the former works described the experiment process without mentioned whether this laser wavelength suitable for this target materials/solvent or not. Getting a better understand of the way that laser wavelength and laser pulse duration 
influence the laser ablation process, film morphology and material structure after deposition is really important for the development of the MAPLE technique. The future research for the MAPLE technique may focus on this part and the cooperation between the laser and the volatile solvent.

\section{Conclusion}

In this article, we briefly discussed origins of MAPLE deposition, the mechanism of the MAPLE deposition process and the application of the MAPLE technique. In about 20 years, MAPLE technique achieved success of depositing various materials such as polymer, protein and nanoparticles. It emerged as a viable alternative route to fabricate thin film without influence the structure and the property of the target material. MAPLE deposited thin film could be applied to drug delivery systems, medical and implant coatings, organic electronic devices and sensors. By changing the surface property of the target, MAPLE technique can remove the drawback of a series of materials or devices and broad the application field of these material and devices. It is clear that the MAPLE still have some disadvantages such as it may change the structure of the materials when the solvent selection is not appropriate or the laser fluence is too high for the target material. This technique is highly depend on the coordinate between the laser parameter, solvent selection and the property of the target material. The future work of MAPLE deposition technique will include more about the solvent selection and the laser parameter influence.

\section{References}

1. Piqué A (2011) The Matrix-Assisted Pulsed Laser Evaporation (MAPLE) process: origins and future directions. Applied Physics A 105: 517-528.

2. Lapshin RV, Alekhin AP, Kirilenko AG, Odintsov SL, Krotkov VA (2010) Vacuum ultraviolet smoothing of nanometer-scale asperities of Poly (methyl methacrylate) surface. Journal of Surface Investigation. X-ray, Synchrotron and Neutron Techniques 4: $1-11$.

3. Alekhin AP, Boleiko GM, Gudkova SA, Markeev AM, et al. (2010) Synthesis of biocompatible surfaces by nanotechnology methods. Nanotechnologies in Russia 5: 696-708.

4. Bertazzo S, Rezwan K (2010) Control of alpha-alumina surface charge with carboxylic acids. Langmuir 26: 3364-3371. [Crossref]

5. Bertazzo S, Zambuzzi WF, Da Silva HA, Ferreira CV, Bertran CA (2009) Bioactivation of alumina by surface modification: a possibility for improving the applicability of alumina in bone and oral repair. COIR 20: 288-293

6. London G, Chen KY, Carroll GT, Feringa BL (2013) Towards Dynamic Control of Wettability by Using Functionalized Altitudinal Molecular Motors on Solid Surfaces. Chemistry - A European Journal 19: 10690-10697.

7. Montero L, Gabriel G, Guimerà A, Villa R, Gleason KK (2012) Increasing biosensor response through hydrogel thin film deposition: Influence of hydrogel thickness. Vacuum 86: 2102-2104.

8. Fan R, Deng X, Zhou L, Gao X, Fan M (2014) Injectable thermosensitive hydrogel composite with surface-functionalized calcium phosphate as raw materials. International Journal of Nanomedicine 9: 615-626.

9. Choy K L (2003) Chemical vapour deposition of coatings. Prog Mater Sci 48: 57-170.

10. Ryu DY, Shin K, Drockenmuller E, Hawker CJ, Russell TP (2005) A generalized approach to the modification of solid surfaces. Science 308: 236-239. [Crossref]

11. Nash ME, Carroll WM, Foley PJ, Maguire G, Connell CO, et al. (2012) Ultra-thin spin coated crosslinkable hydrogels for use in cell sheet recovery-synthesis, characterisation to application. Soft Matter 8: 3889-3899.

12. Hume PS, Bowman CN, Anseth KS (2011) Functionalized PEG hydrogels through reactive dip-coating for the formation of immunoactive barriers. Biomaterials 32 : 6204-6212. [Crossref]

13. Ma J, Sahai Y (2012) A Direct Borohydride Fuel Cell with Thin Film Anode and Polymer Hydrogel Membrane. ECS Electrochem Lett 1: F41-F43.
14. Caricato AP, Capone S, Epifani M, Lomascolo M, Luches A, et al. (2008) In Nanoparticle thin films deposited by MAPLE for sensor applications $69850 \mathrm{H}-69850 \mathrm{H}-13$.

15. Piqué A, McGill R A, Chrisey DB, Leonhardt D, Mslna TE, et al. (1999) Growth of organic thin films by the matrix assisted pulsed laser evaporation (MAPLE) technique. Thin Solid Films 355-356, 536-541.

16. Smith HM, Turner AF (1965) Vacuum Deposited Thin Films Using a Ruby Laser. Appl Opt 4: 147-148.

17. Hansen SG, Robitaille TE (1988) Formation of polymer films by pulsed laser evaporation. Appl Phys Lett 52: 81-83.

18. Hansen SG, Robitaille TE (1988) Arrival time measurements of films formed by pulsed laser evaporation of polycarbonate and selenium. Journal of Applied Physics 64: 21222129.

19. McGill R, Chrisey D (2000) US Patent, 6,025,036. February $15^{\text {th }}$.

20. Piqué A, Chrisey DB, Spargo BJ, Bucaro MA, Vachet RW, et al. (1998) Use of Matrix Assisted Pulsed Laser Evaporation (Maple) for the Growth of Organic Thin Films. MRS Proceedings 526.

21. Bloisi F, Cassinese A, Papa R, Vicari L, Califano V (2008) Matrix-Assisted Pulsed Laser Evaporation of polythiophene films. Thin Solid Films 516: 1594-1598.

22. Sima F, Davidson P, Pauthe E, Sima LE, Gallet O, et al. (2011) Fibronectin layers by matrix-assisted pulsed laser evaporation from saline buffer-based cryogenic targets. Acta Biomater 7: 3780-3788.

23. Caricato AP, Luches A (2011) Applications of the matrix-assisted pulsed laser evaporation method for the deposition of organic, biological and nanoparticle thin films: a review. Applied Physics A 105: 565-582.

24. McGill RA, Chrisey DB, Pique A, Mlsna TE (1998) In Matrix-assisted pulsed-laser evaporation (MAPLE) of functionalized polymers: applications with chemical sensors: 255-266.

25. McGill RA, Chung R, Chrisey DB, Dorsey PC, Matthews P, et al. (1998) Performance optimization of surface acoustic wave chemical sensors. IEEE Transactions on Ultrasonics, Ferroelectrics, and Frequency Control 45: 1370-1380.

26. Ringeisen BR, Callahan J, Wu PK, Piqué A, Spargo B, McGill, RA, et al. (2001) Novel Laser-Based Deposition of Active Protein Thin Films. Langmuir 17: 3472-3479.

27. Wu PK, Ringeisen BR, Callahan J, Brooks M, Bubb DM, et al. (2001) The deposition, structure, pattern deposition, and activity of biomaterial thin-films by matrix-assisted pulsed-laser evaporation (MAPLE) and MAPLE direct write. Thin Solid Films 607614.

28. Toftmann B, Papantonakis MR, Auyeung RCY, Kim W, O’Malley SM, et al. (2004) UV and RIR matrix assisted pulsed laser deposition of organic MEH-PPV films. Thin Solid Films 453-454, 177-181.

29. Gutiérrez-Llorente A, Horowitz G, Pérez-Casero R, Perrière J, et al. (2004) Growth of polyalkylthiophene films by matrix assisted pulsed laser evaporation. Organic Electronics 5: 29-34.

30. Piqué A, Auyeung RCY, Stepnowski JL, Weir DW, Arnold CB, et al. (2003) Laser processing of polymer thin films for chemical sensor applications. Surface and Coatings Technology 163-164, 293-299.

31. Houser EJ, Chrisey DB, Bercu M, Scarisoreanu ND, Purice A, et al. (2006) Functionalized polysiloxane thin films deposited by matrix-assisted pulsed laser evaporation for advanced chemical sensor applications. Appl Surf Sci 252: 4871-4876.

32. Piqué A, Wu P, Ringeisen BR, Bubb DM, Melinger JS, et al. (2002) Processing of functional polymers and organic thin films by the matrix-assisted pulsed laser evaporation (MAPLE) technique. Appl Surf Sci 186: 408-415.

33. Chrisey DB, Piqué A, McGill RA, Horwitz JS, Ringeisen BR, et al. (2003) Laser deposition of polymer and biomaterial films. Chem Rev 103: 553-576. [Crossref]

34. Sellinger AT, Leveugle EM, Gogick K, Zhigilei LV, Fitz-Gerald JM, et al. (2006) Laser processing of polymer nanocomposite thin films. Journal of Vacuum Science \& Technology A 24: 1618-1622.

35. Cristescu R, Mihaiescu D, Socol G, Stamatin I, Mihailescu IN, et al. (2004) Deposition of biopolymer thin films by matrix assisted pulsed laser evaporation. Applied Physics A 79: 1023-1026.

36. Mercado AL, Allmond CE, Hoekstra JG, Fitz-Gerald JM (2005) Pulsed laser deposition vs. matrix assisted pulsed laser evaporation for growth of biodegradable polymer thin films. Applied Physics A 81: 591-599. 
37. Bubb D, Wu P, Horwitz J, Callahan J, Galicia M. Vertes A (2002) The effect of the matrix on film properties in matrix-assisted pulsed laser evaporation. Journal of applied physics 91:2055-2058

38. Rodrigo K, Toftmann B, Schou J, Pedrys R (2004) Laser-induced ion emission during polymer deposition from a flash-frozen water ice matrix. Chemical Physics Letters 399: 368-372.

39. Popescu C, Roqueta J, Pérez del Pino A, Moussaoui M, Nogués MV, et al. (2011) Processing and immobilization of enzyme Ribonuclease A through laser irradiation. $J$ Mater Res 26: 815-821.

40. Sima F, Mutlu EC, Eroglu MS, Sima LE, Serban N, et al. (2011) Levan nanostructured thin films by MAPLE assembling. Biomacromolecules 12: 2251-2256. [Crossref]

41. Cristescu R, Popescu C, Socol G, Visan A, Mihailescu IN, et al. (2011) Deposition of antibacterial of poly(1,3-bis-(p-carboxyphenoxy propane)-co-(sebacic anhydride)) 20:80/ gentamicin sulfate composite coatings by MAPLE. Appl Surf Sci 257: 5287-5292.

42. Miroiu FM, Socol G, Visan A, Stefan N, Craciun D, et al. (2010) Composite biocompatible hydroxyapatite-silk fibroin coatings for medical implants obtained by Matrix Assisted Pulsed Laser Evaporation. Materials Science and Engineering B 169: 151-158.

43. Floroian L, Florescu M, Sima F, Popescu-Pelin G, Ristoscu C, et al. (2012) Synthesis of biomaterial thin films by pulsed laser technologies: Electrochemical evaluation of bioactive glass-based nanocomposite coatings for biomedical applications. Materials Science and Engineering: C 32: 1152-1157.

44. Sima F, Davidson P, Pauthe E, Gallet O, Anselme K, et al. (2011) Thin films of vitronectin transferred by MAPLE. Applied Physics A 105: 611-617.

45. Paun IA, Ion V, Luculescu CR, Dinescu M, Canulescu S, et al. (2012) In vitro studies of PEG thin films with different molecular weights deposited by MAPLE. Applied Physics A 109: 223-232.

46. Pate R, McCormick R, Chen L, Zhou W, Stiff-Roberts AD (2011) RIR-MAPLE deposition of conjugated polymers for application to optoelectronic devices. Applied Physics A 105: 555-563.

47. Caricato AP, Luches A, Leggieri G, Martino M, Rella R (2012) Matrix-assisted pulsed laser deposition of polymer and nanoparticle films. Vacuum 86: 661-666.

48. Park HK, Schriver KE, Haglund RF (2011) Resonant infrared laser deposition of polymer-nanocomposite materials for optoelectronic applications. Applied Physics A 105: 583-592.

49. McCormick RD, Lenhardt J (2012) Stiff-Roberts, A. D., Effects of Emulsion-Based Resonant Infrared Matrix Assisted Pulsed Laser Evaporation (RIR-MAPLE) on the Molecular Weight of Polymers. Polymers 4: 341.

50. Caricato AP, Cesaria M, Gigli G, Loiudice A, Luches A, et al. (2012) Poly-(3hexylthiophene)/[6,6]-phenyl-C61-butyric-acid-methyl-ester bilayer deposition by matrix-assisted pulsed laser evaporation for organic photovoltaic applications. Appl Phys Lett 100: 073306.

51. Adil D, Ukah NB, Gupta RK, Ghosh K, Guha S (2010) Interface-controlled pulsedlaser deposited polymer films in organic devices. Synth Met 160: 2501-2504.

52. Ukah NB, Adil D, Granstrom J, Gupta RK, Ghosh K, Guha S (2011) Matrix-assisted pulsed-laser evaporated polymer films in all-organic field-effect transistors and metalinsulator-semiconductor diodes. Organic Electronics 12: 1580-1587.

53. Gupta R, Ghosh KS, Guha D, Adil NB Ukah (2011) Appl Phys A 105: 547-554.

54. McCormick RD, Cline ED, Chadha AS, Zhou W, et al. (2012) In Tuning the refractive index of blended polymer films by RIR-MAPLE deposition 825806-825806-6.
55. Yijun S, Takashi E, Chunlin S, Liangying Z, Xi Y (2004) Formation Mechanism for High-Surface-Area Anatase Titania Nanoparticles Prepared by Metalorganic Chemical Vapor Deposition. JaJAP 43: 3544.

56. Yuan W, Masaki T, Kazuo F (1999) High Resolution Transmission Electron Microscopy Study on the Structure of Ge Nanoparticles by Using an Ultrahigh Vacuum-Molecular Beam Epitaxy-Transmission Electron Microscope System. JaJAP 38: 7241.

57. Wu PK, Fitz-Gerald J, Pique A, Chrisey DB, McGill RA (2000) Deposition Of Nanotubes and Nanotube Composites Using Matrix-Assisted Pulsed Laser Evaporation. MRS Proceedings 617.

58. Caricato AP, Luches A, Rella R (2009) Nanoparticle thin films for gas sensors prepared by matrix assisted pulsed laser evaporation. Sensors (Basel) 9: 2682-2696. [Crossref]

59. Pérez del Pino Á, György E, Cabana L, Ballesteros B, Tobias G (2012) Deposition of functionalized single wall carbon nanotubes through matrix assisted pulsed laser evaporation. Carbon 50: 4450-4458.

60. Hunter CN, Check MH, Bultman JE, Voevodin AA (2008) Development of matrixassisted pulsed laser evaporation (MAPLE) for deposition of disperse films of carbon nanoparticles and gold/nanoparticle composite films. Surface and Coatings Technology 203: 300-306.

61. György E, Pino AP, Roqueta J, Ballesteros B, Miguel, et al. (2011) Synthesis and Laser Immobilization onto Solid Substrates of $\mathrm{CdSe} / \mathrm{ZnS}$ Core-Shell Quantum Dots. The Journal of Physical Chemistry C 115: 15210-15216.

62. Pérez del Pino A, György E, Logofatu C, Puigmartí-Luis J, et al. (2015) Laser-induced chemical transformation of graphene oxide-iron oxide nanoparticles composites deposited on polymer substrates. Carbon 93: 373-383.

63. Cristescu R, Popescu C, Socol G, Iordache I, Mihailescu IN, et al. (2012) Magnetic core/shell nanoparticle thin films deposited by MAPLE: Investigation by chemical, morphological and in vitro biological assays. Appl Surf Sci 258: 9250-9255.

64. Pozuelo J, Compañ V, González-Méijome JM, González M, Mollá S (2014) Oxygen and ionic transport in hydrogel and silicone-hydrogel contact lens materials: An experimental and theoretical study. J Membr Sci 452: 62-72.

65. Iskeleli G, Karakoc Y, Ozkok A, Arici C, Ozcan O, et al. (2013) Comparison of the effects of first and second generation silicone hydrogel contact lens wear on tear film osmolarity. International Journal of Ophthalmology 6: 666-670.

66. Kodjikian L, Casoli-Bergeron E, Malet F, Janin-Manificat H, Freney J, et al. (2008) Bacterial adhesion to conventional hydrogel and new silicone-hydrogel contact lens materials. Graefe's Archive for Clinical and Experimental Ophthalmology 246: 267273.

67. Thissen H, Gengenbach T, du Toit R, Sweeney DF, Kingshott P, et al. (2010) observations of biofouling on PEO coated silicone hydrogel contact lenses. Biomaterials 31: 5510-5519.

68. Yin P, Huang GB, Tse WH, Bao YG, Denstedt J, et al. (2015) Nanocomposited silicone hydrogels with a laser-assisted surface modification for inhibiting the growth of bacterial biofilm. Journal of Materials Chemistry B 3:3234-3241.

69. Huang G, Chen Y, Zhang J (2016) Nanocomposited coatings produced by laser-assisted process to prevent silicone hydogels from protein fouling and bacterial contamination. Appl. Surf. Sci. 360: 383-388.

70. Huang G, Tse WH, Zhang J (2016) Deposition of a hydrophilic nanocomposite-based coating on silicone hydrogel through a laser process to minimize UV exposure and bacterial contamination. RSC Advances 6: 67166-67172.

Copyright: C2017 Yang S. This is an open-access article distributed under the terms of the Creative Commons Attribution License, which permits unrestricted use, distribution, and reproduction in any medium, provided the original author and source are credited. 